University of Nebraska - Lincoln

DigitalCommons@University of Nebraska - Lincoln

1978

Studies of the Temporary Anion States of Unsaturated

Hydrocarbons by Electron Transmission Spectroscopy

Kenneth D. Jordan

Paul Burrow

Follow this and additional works at: https://digitalcommons.unl.edu/physicsburrow

Part of the Atomic, Molecular and Optical Physics Commons

This Article is brought to you for free and open access by the Research Papers in Physics and Astronomy at DigitalCommons@University of Nebraska - Lincoln. It has been accepted for inclusion in Paul Burrow Publications by an authorized administrator of DigitalCommons@University of Nebraska - Lincoln. 


\title{
Studies of the Temporary Anion States of Unsaturated Hydrocarbons by Electron Transmission Spectroscopy
}

\author{
Kenneth D. Jordan \\ Mason Laboratory, Department of Engineering and Applied Science, \\ Yale University, New Haven, Connecticut 06520 \\ Paul D. Burrow \\ Behlen Laboratory of Physics, University of Nebraska, \\ Lincoln, Nebraska 68588
}

The concept of occupied and unoccupied orbitals has provided a useful means for visualizing many of the most important properties of molecular systems. Yet, there is a curious imbalance in our experimental knowledge of the energies of occupied and unoccupied orbitals. Whereas photoelectron spectroscopy has provided a wealth of data on positive ion states and has established that they can be associated, within the context of Koopmans' theorem, with the occupied orbitals of the neutral molecule, the corresponding information for the negative ion states, associated with the normally unoccupied orbitals, is sparse. In part this reflects the experimental difficulties connected with measuring the electron affinities of molecules which possess stable anions. A more interesting aspect, in our view, is that for many

Published in Accounts of Chemical Research Vol. 11, 1978, pp 341-348

Copyright (C) 1978 American Chemical Society. Used by permission.

Submitted September 2, 1977. 
molecules even the ground state of the anion lies above the ground state of the neutral molecule and thus is unstable with respect to autodetachment of the additional electron. The temporary anions which we will discuss in this Account possess lifetimes in the range from $10^{-}$ 12 to $10^{-15} \mathrm{~s}$ in the gas phase. Ephemeral as these anions might appear, their existence is amply demonstrated in electron-scattering experiments. At energies in which an impacting electron may be temporarily captured into a normally unoccupied orbital, the electron-scattering cross section undergoes a pronounced change in magnitude, commonly referred to as a "resonance" by those in the scattering field. ${ }^{1}$

A number of electron-scattering techniques may be used to investigate temporary anion formation. In this article we will describe only one, the electron transmission method in the format devised by Sanche and Schulz. ${ }^{2}$ This technique has numerous assets: it is both conceptually and experimentally straightforward, and it is likely the most sensitive means for detection of temporary anions.

During the last few years, electron transmission spectroscopy has seen increasing application to organic molecules. In the following sections, we discuss our results and those of other workers which bear on the characteristics of the temporary anion states associated with lowlying $\pi^{*}$ orbitals in a number of classes of hydrocarbons. In addition we will describe briefly the relationship of this work to other chemical studies, such as optical absorption measurements on anions in solution, molecular orbital theories of reactivity, and studies of low-lying electronic states of neutral molecules.

\section{Temporary Anion Formation}

A conceptually useful picture of temporary anion formation has been developed from studies on atoms and diatomic molecules. ${ }^{1}$ The essential ideas may be carried over to the hydrocarbons we will discuss here. Let us first consider electron scattering from the spherically symmetric potential field of an atom. The incident beam of electrons may be represented as a plane wave containing all components of angular momentum. If the atom possesses an energetically accessible unfilled orbital, characterized by a particular value of the orbital angular momentum quantum number $l$, then an incident electron with this 


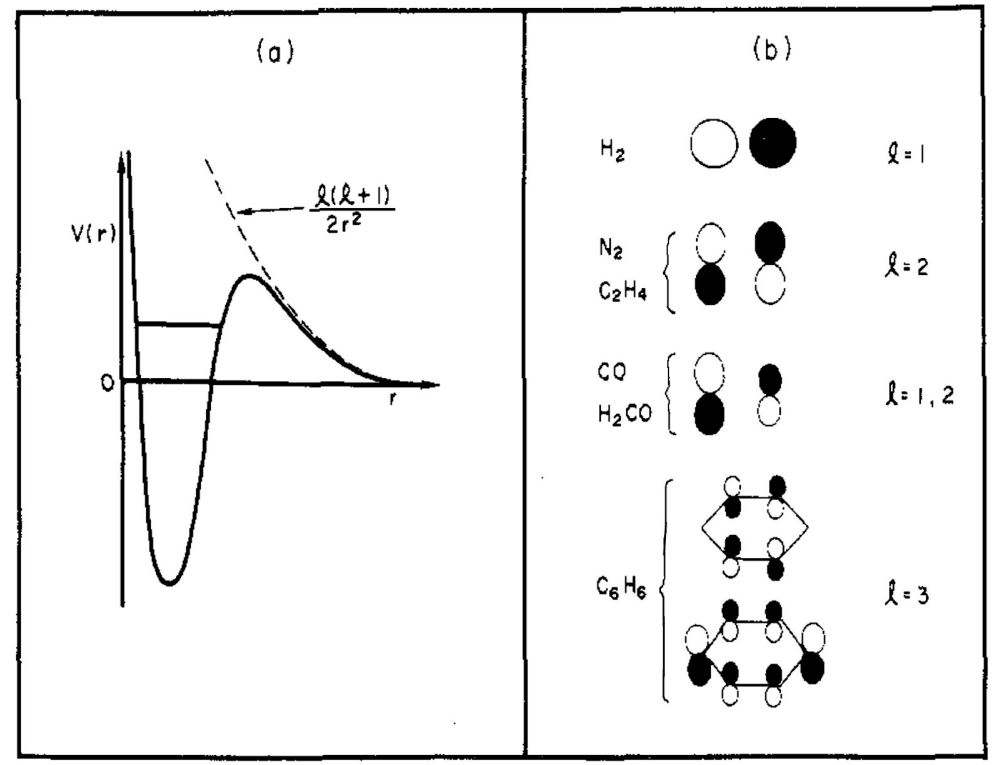

Figure 1. (a) The potential energy of an electron with angular momentum $l$ in the field of an atom as a function of the electron- atom separation; (b) Sketches of the LUMOs of several simple molecules and their important angular momentum components.

appropriate orbital symmetry may be temporarily "captured". Both the energy and lifetime of such an anionic state are determined by the total potential in which the electron moves. In Figure 1a we illustrate the general form of the potential. At large separation the electron- atom interaction consists of two important terms, an attractive polarization potential and the repulsive centrifugal potential associated with the angular momentum of the electron. These two terms in general combine to form a barrier. At closer distances, a pronounced dip is produced by the short-range screened Coulomb forces.

The energy of the anion state is largely determined by the strength of the attractive portion of the potential. The lifetime of the temporary anion is a function both of the size of the barrier, which is strongly dependent on $l$ as seen in Figure 1a, and on the anion energy since the combination of these factors determines the relative height and thickness of the barrier through which the electron must tunnel in order to detach.

In the mechanism outlined here, the electron is temporarily bound because of the "shape" of the potential field it experiences, and the 
resulting structure in the electron-scattering cross section is generally referred to as a shape resonance. Historically, such resonances in electron-molecule scattering have been known for almost 20 years. ${ }^{1}$ Surprisingly, the corresponding process in atoms has only recently been studied. ${ }^{3}$

The extension of the shape resonance picture to molecules is more complex because of the noncentral potential in which the electron moves. Each unoccupied orbital of the target molecule has a characteristic charge distribution whose symmetry determines the possible angular momentum components which may be "captured". 4 The relative contribution of these components will be determined by the detailed shape of the orbital. For molecules with a high degree of symmetry, only the lowest few allowed components will be necessary to describe the scattering behavior. These important angular momentum components can be visualized by expanding the orbital charge distribution in spherical harmonics. In Figure 1b, sketches of the lowest unfilled molecular orbitals (LUMOs) are given for several simple molecules along with the dominant angular momentum components.

In more complicated molecules with low symmetry, this simple approach is less obvious because of the greater number of angular momentum components needed to describe the scattering process. Nevertheless we have found these notions to be surprisingly useful for obtaining a qualitative understanding of both the energies and relative lifetimes of temporary anions.

\section{Electron Transmission Spectroscopy}

A number of techniques may be used to detect the resonant variation in the electron scattering cross section which occurs at those energies at which a temporary anion is formed. In an electron transmission method, a signal is derived which is proportional to the total electron scattering cross section, that is, the sum over all elastic and inelastic scattering processes. Thus the technique has great inherent sensitivity to sharp structure arising from temporary anion formation. A transmission experiment is quite simple conceptually. An electron monochromator injects a beam of current $I_{\mathrm{o}}$ into a scattering chamber 
containing gas at density $N$ whose cross section for electron scattering is $Q(E)$, a function of electron energy. After traversing a path length $L$, the scattered portion of the incident beam is rejected. The current "transmitted" to the collector is the unscattered current given by $I_{\mathrm{O}}$ $\exp (-N Q L)$. A signal is thereby derived which is related to the total scattering cross section as a function of electron energy.

The visibility of small variations in the cross section may be increased by means of a modification introduced by Sanche and Schulz. ${ }^{2}$ The energy of the impacting electrons is modulated by applying a small ac voltage to an electrode contained within the collision chamber and the ac component of the transmitted current is detected synchronously. The resulting signal is proportional to the derivative with respect to energy of the transmitted current. The rapid variations in the cross section, which are due to temporary anion formation, are thereby greatly enhanced with respect to the broad, slowly varying features which result from nonresonant scattering.

Although temporary anions of hydrocarbons have been observed using direct electron transmission without modulation, as in the work of Hasted and co-workers, ${ }^{5}$ the full potential of the method was not exploited until the introduction of the derivative technique. ${ }^{2}$ Studies of rare gases, di- and triatomic molecules, and ethylene and benzene were carried out by Sanche and Schulz, ${ }^{2-6}$ and of several azines by $\mathrm{Ne}-$ nner and Schulz, 7 in an exemplary series of papers.

\section{Anion States and the Transmission Spectrum}

In Figure 2, we illustrate the connection between the anion and neutral states and the structure observed in a transmission experiment. In the upper left portion of Figure 2, potential curves are shown for a hypothetical diatomic molecule $\mathrm{AB}$ and its anion $\mathrm{AB}^{-}$. For convenience we assume that the anion lives long enough to possess well-defined vibrational levels. For those energies at which the electron attaches to $\mathrm{AB}$, that is, occupies the appropriate normally unfilled molecular orbital, transitions into the vibrational levels of $\mathrm{AB}^{-}$take place. At the energy of each level, the total scattering cross section is sharply enhanced by the resonant capture of the electron. The resulting cross section is shown in the upper right of Figure 2 as a progression of 


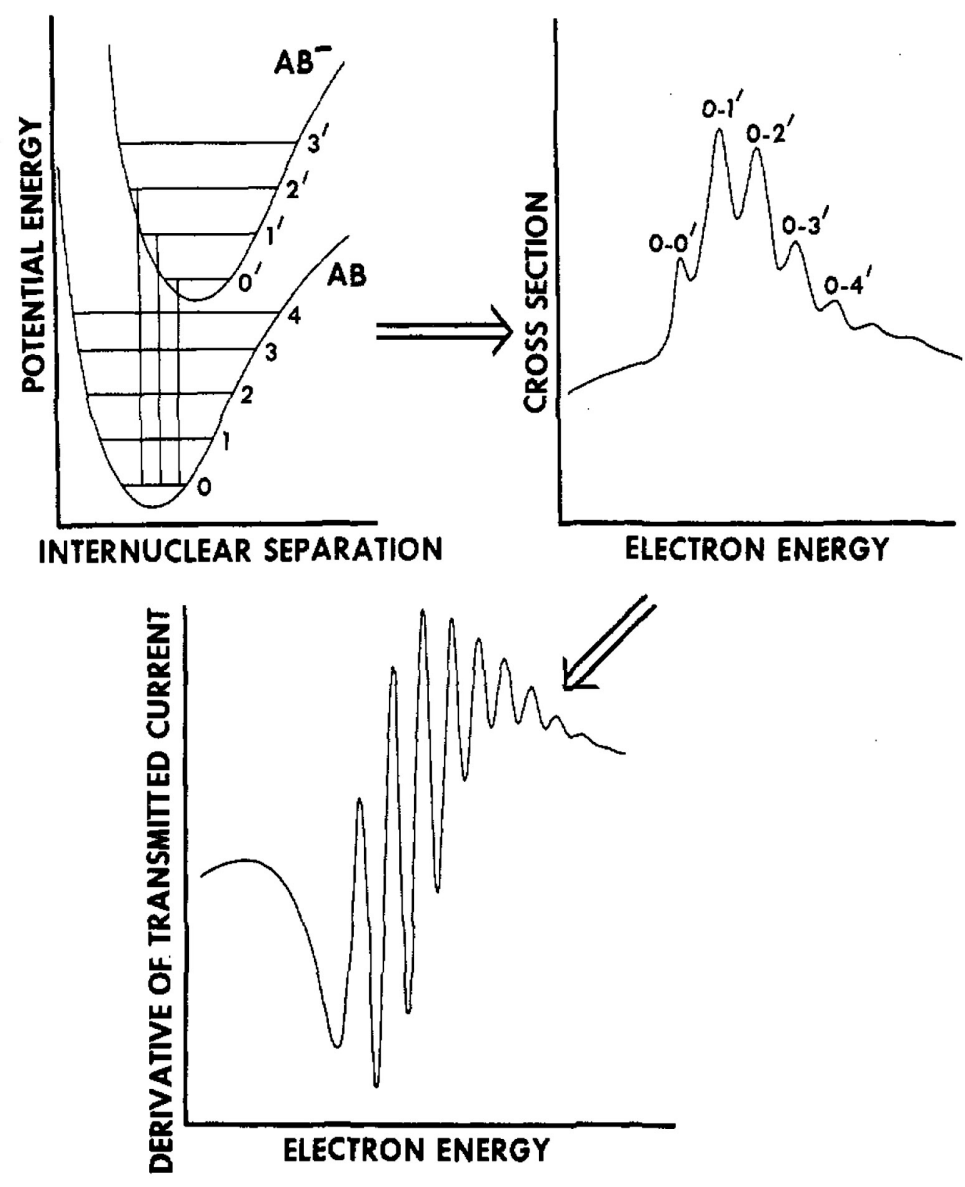

Figure 2. (Upper left) Potential energy curves for a hypothetical diatomic $A B$ and its temporary anion $\mathrm{AB}^{-}$; (upper right) the cross section for electron scattering from $\mathrm{AB}$; lower, the derivative of transmitted current as a function of electron energy.

vibrational peaks, characterized by the appropriate Franck-Condon factors, superposed on a smoothly varying contribution from the nonresonant scattering processes.

The corresponding transmission spectrum resembles that shown at the bottom of Figure 2 in the standard format, that is, with the derivative of the transmitted current plotted as a function of impact energy. If the transition to the ground vibrational level of a given anion state can be identified, then the adiabatic electron affinity (EA) associated with that state can be determined. If the o-o transition cannot be identified or if there is no vibrational structure, only the most probable transition energy or vertical EA can be ascertained. 
The existence of vibrational structure in the transmission spectrum depends directly on the lifetime of the anion. Roughly speaking, if the average lifetime is longer than the period ${ }^{8}$ of the nuclear motion, then well-developed structure appears which may be associated with vibrational motion of the anion in the usual sense. For shorter lifetimes, the widths of the resonant peaks are broadened as prescribed by the uncertainty principle, and the visibility of the structure is therefore lessened. The nuclear motion of the anion may be described by a wavepacket whose amplitude is continuously shrinking due to the autodetachment of the electron. Birtwistle and Herzenberg ${ }^{9}$ have shown that structure appears in the scattering cross section if the lifetime of the anion permits a portion of the nuclear wavepacket to survive the traversal of the potential surface once and be reflected from the other side. The resulting interference structure is not due to true vibrational motion of the anion but can be referred to as "quasi-vibrational" in origin.

In Figure 3 we display the transmission spectra of four molecules: nitrogen, carbon monoxide, ethylene, and formaldehyde. This series was chosen to illustrate that one can obtain qualitatively useful information about the lifetimes of temporary molecular anions from the considerations of energy and angular momentum discussed earlier.

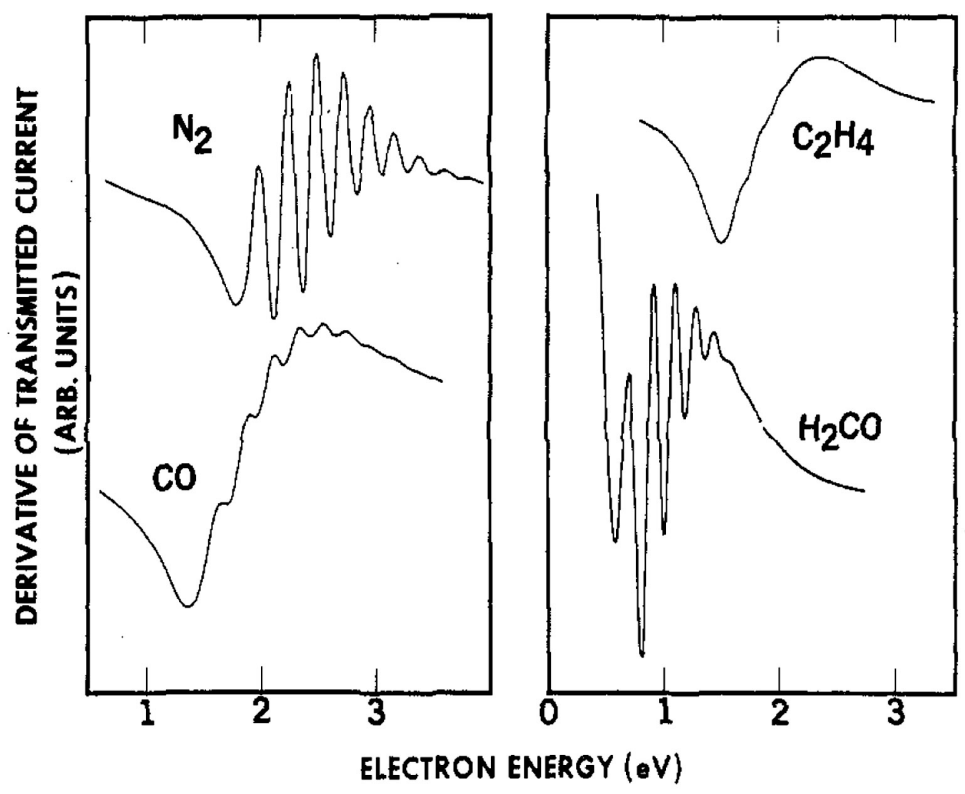

Figure 3. The derivative of transmitted current as a function of electron energy in $\mathrm{N}_{2}, \mathrm{CO}, \mathrm{C}_{2} \mathrm{H}_{4}$, and $\mathrm{H}_{2} \mathrm{CO}$. 
Although the ground state of $\mathrm{CO}^{-}$lies slightly below that of $\mathrm{N}_{2}{ }^{-}$, the vibrational structure in the transmission spectrum of $\mathrm{CO}$ is much less pronounced than in $\mathrm{N}_{2}$. This may be understood ${ }^{10}$ from an examination of the partial wave expansions of the respective LUMOs: the lowest component in the $\mathrm{N}_{2}$ LUMO is $l=2$, while the LUMO of CO has sizable contributions from both $l=1$ and $l=2$. The additional electron tunneling in the $l=1$ "channel", which takes place through a smaller barrier, thus shortens the lifetime of $\mathrm{CO}^{-}$relative to that of $\mathrm{N}_{2}{ }^{-}$.

The second pair of molecules, ethylene and formaldehyde, possess LUMOs characterized by the same $l$ values as those of nitrogen and carbon monoxide, respectively, as seen in Figure lb. One might expect therefore that formaldehyde would display even weaker structure than ethylene. As shown in Figure 3, the opposite is true. In this case, the ground state of $\mathrm{H}_{2} \mathrm{CO}^{-}$lies considerably below that of $\mathrm{C}_{2} \mathrm{H}_{4}{ }^{-}$, illustrating that a sufficiently low anion energy can more than compensate for a smaller barrier. The anion lifetime depends on both the barrier height and thickness seen by the autodetaching electron.

Although electron transmission spectroscopy is analogous in many ways to photoelectron spectroscopy, a notable difference exists in the lifetime of the ionic species. The short anion lifetimes create special problems for a theoretical description of the nuclear motion, and indeed, a general theoretical treatment of anion lifetimes has not yet been developed. Because of the intimate relationship between the characteristics of the unfilled orbital and the anion lifetime, it seems clear that an understanding of lifetime effects will lead to valuable information about orbital charge distributions. We have found that the lifetimes are very sensitive to substituent effects. For example, the EAs of benzene and toluene differ by less than $0.03 \mathrm{eV}$, yet the vibrational structure in the toluene spectrum ${ }^{11}$ is greatly reduced from that in benzene, suggesting that the lifetime may be shortened by more than a factor of two.

Space does not permit more than a cursory mention of another aspect of anion lifetimes. Temporary anions play a role of profound importance in the vibrational excitation of molecules by electron impact. ${ }^{12}$ Upon ejection of the electron from the anion, the molecule will generally be left in one of the vibrational levels of the ground electronic state. The distribution of such vibrational levels in the neutral molecule will depend on the extent to which the molecule is distorted from its equilibrium geometry by the "additional" electron. This is 
clearly a function of the anion lifetime. The specific vibrational modes which are excited in this process depend, of course, on the symmetry of the orbital. This has been nicely demonstrated by Wong and Schulz ${ }^{13}$ in their study of the vibrational excitation of benzene.

\section{Electron Transmission Studies in Hydrocarbons}

Ethylene. Our interest in the anions of hydrocarbons was stimulated in part by a reexamination of the transmission spectrum of ethylene, the simplest unsaturated hydrocarbon. The ground state of the ethylene anion, formed by the capture of an incident electron into the low-lying $b_{2 g} \pi^{*}$ orbital, was first observed in electron transmission by Boness et al. ${ }^{5}$ and later by Sanche and Schulz. ${ }^{6}$ Unlike previous investigators, we observed ${ }^{14}$ weak undulations superposed on the characteristic resonance profile, as seen in Figure 3. We assigned this structure to motion of the anion along the C-C symmetric stretch coordinate. The detection of the faint quasivibrational structure was valuable in two respects. First, it enabled us to determine the previously unknown adiabatic EA of ethylene within reasonable error limits. Second, the structure provided us with a lifetime "benchmark" for the resonance associated with an unperturbed $\mathrm{C}-\mathrm{C}$ double bond as well as an example of a lifetime which is just sufficiently long for detectable structure to appear in the transmission spectrum. Substituent effects on this lifetime are quite pronounced. For example, although the propene anion is only $0.21 \mathrm{eV}$ less stable than the ethylene anion, ${ }^{15}$ the lowering of symmetry due to methyl substitution and the accompanying admixture of lower $l$ values result in a complete loss of quasivibrational structure.

Cyclic Dienes. We turn now to molecules composed of interacting ethylenic groups and demonstrate that electron transmission spectroscopy can be employed to study the splitting between the anion states which results from the coupling of the ethylenic $\pi^{*}$ orbitals.

Two useful concepts which have been introduced to explain the interaction between functional units are through-space and throughbond coupling. ${ }^{16}$ For example, in cyclic dienes, the splitting between the two occupied $\pi$ orbitals depends on the separation and orientation of the two double bonds, that is, on through-space interaction, 
as well as on through-bond coupling due to the interaction with the methylenic pseudo- $\pi$ orbitals. The molecules 1,4-cyclohexadiene and norbornadiene have played a particularly interesting role in the studies of the relative importance of these two effects using photoelectron spectroscopy. The through-space interaction of the two ethylenic $\pi$ orbitals of these molecules gives rise to bonding and antibonding molecular orbitals, $\pi_{\mathrm{b}}$ and $\pi_{\mathrm{a}}$. In the absence of interactions other than direct through-space coupling, the $\pi_{\mathrm{b}}$ orbital will be more stable than the $\pi_{\mathrm{a}}$. However, from photoelectron studies, it has been established that the reverse is true for 1,4-cyclohexadiene. The reason for this, as elegantly demonstrated by Batich et al. ${ }^{17}$ and Hoffmann et al., ${ }^{18}$ is that the pseudo- $\pi$ orbitals of the $\mathrm{CH}_{2}$ groups push the $\pi_{b}$ orbital above the $\pi_{\mathrm{a}}$ orbital.

On the other hand, we have suggested ${ }^{15}$ that the ordering of the anion states of 1,4-cyclohexadiene is normal, that is, with $\pi_{\mathrm{b}}$ * below $\pi_{\mathrm{a}}{ }^{*}$, since through-bond coupling with the $\mathrm{CH}_{2}-\pi$ destabilizes the $\pi_{\mathrm{b}}{ }^{*}$ orbital much less than it does the $\pi_{\mathrm{b}}$ orbital. In support of this claim,

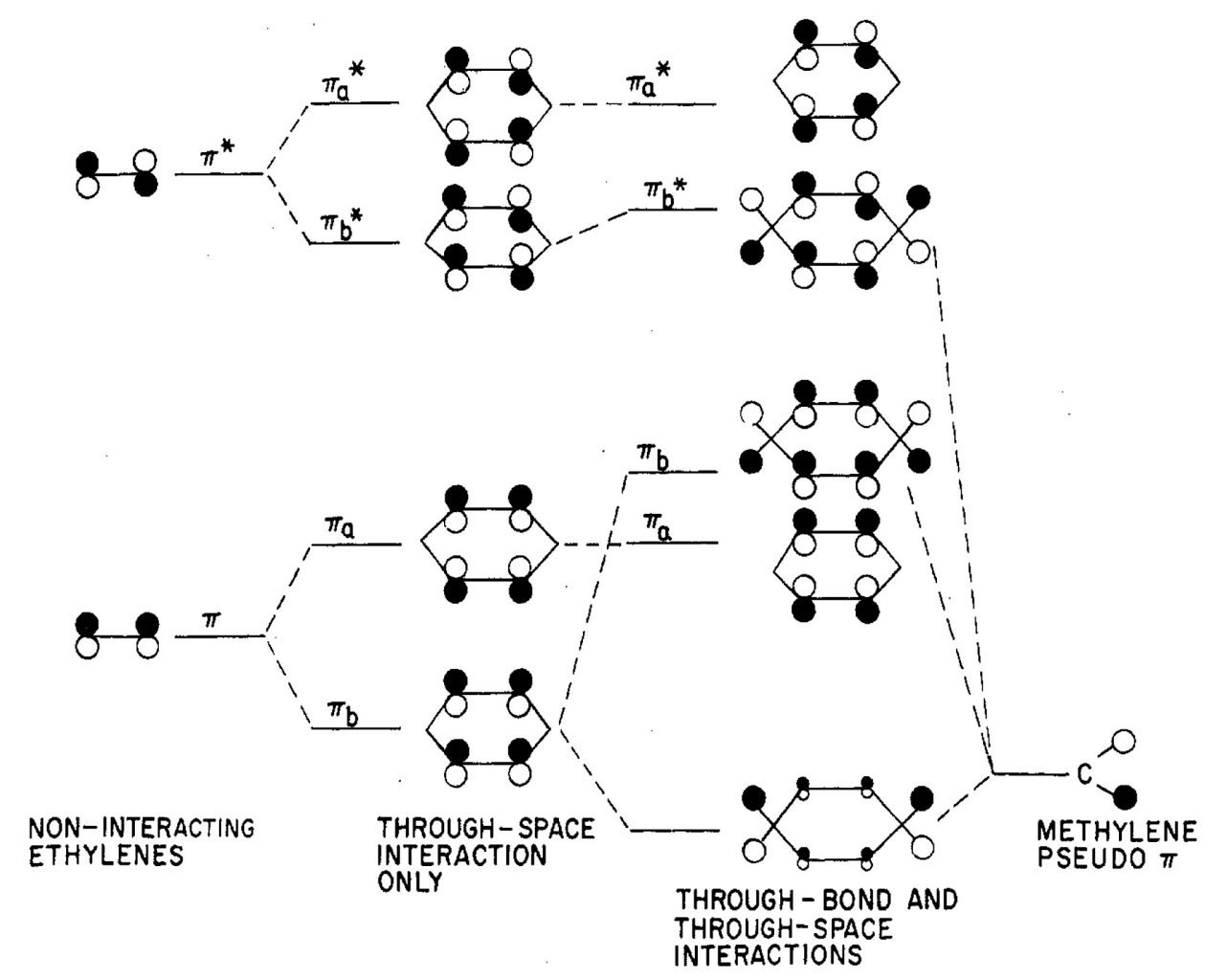

Figure 4. Correlation diagram for 1,4-cyclohexadiene illustrating the interactions between the ethylenic $\pi$ and $\pi^{*}$ orbitals and the methylene pseudo- $\pi$ orbital. 
we note that methyl substitution destabilizes the anion of ethylene by only $0.21 \mathrm{eV}$ while the cation is stabilized by $0.78 \mathrm{eV}$. The interactions between the ethylenic $\pi$ and $\pi^{*}$ orbitals of 1,4-cyclohexadiene with and without the through-bond coupling due to the $\mathrm{CH}_{2}-\pi$ orbitals are depicted in Figure 4.

In norbornadiene, where through-bond coupling is not as important, both the $\pi$ and $\pi^{*}$ orbitals have normal ordering with the $\pi_{\mathrm{b}}$ orbital lying below the $\pi_{\mathrm{a}}$, and $\pi_{\mathrm{b}}{ }^{*}$ lying below $\pi_{\mathrm{a}}{ }^{*}$. As shown in Figure 5 which displays the transmission spectra of 1,4-cyclohexadiene and norbornadiene, the ground anionic state of 1,4-cyclohexadiene is indeed destabilized relative to that of norbornadiene while the first excited anion states occur at nearly the same energy.

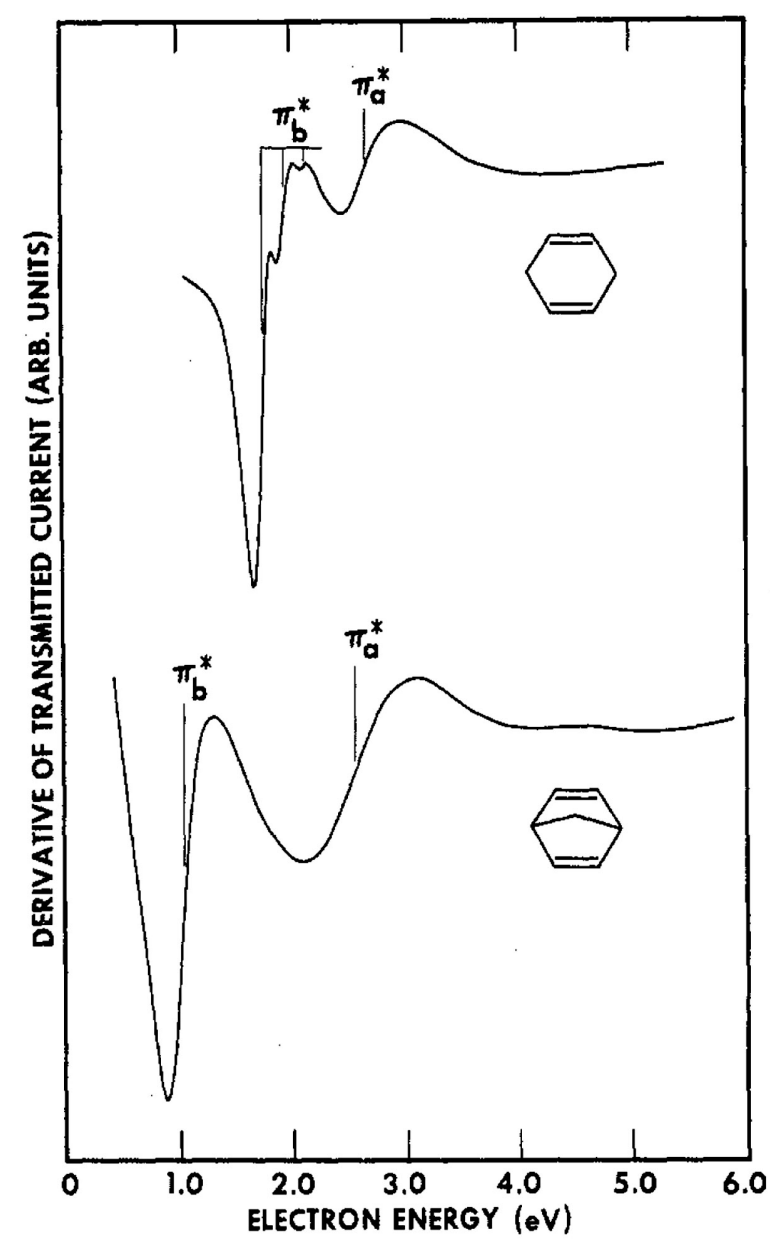

Figure 5. The derivative of transmitted current as a function of electron energy in 1,4-cyclohexadiene (upper) and norbornadiene (lower). Reprinted with permission from ref 15. Copyright 1976 North-Holland Publishing Co. 
Benzene and Substituted Benzenes. Benzene and substituted benzenes have been the subject of numerous studies employing optical and photoelectron spectroscopy. Not surprisingly, several transmission studies have already appeared dealing with the anion states of benzene and substituted benzenes..$^{-7,11,19}$ One of the reasons for the interest in benzene and its derivatives is that both the $\operatorname{HOMO}\left(\mathrm{e}_{1 \mathrm{~g}}\right)$ and LUMO $\left(\mathrm{e}_{2 \mathrm{u}}\right)$ of benzene are degenerate and may be split, for example upon monosubstitution. Moreover, the ground anion and cation states of benzene are examples of Jahn-Teller systems, and many of the alkyl-substituted benzenes display pseudo-Jahn-Teller effects.

Figure 6 shows a correlation diagram of the cation and anion states, as determined by photoelectron and electron transmission spectroscopy, respectively, for benzene, fluoro-, chloro-, and bromobenzenes, phenol, anisole, aniline, and toluene. The splitting of the $\mathrm{e}_{1 \mathrm{~g}}(\pi)$ orbitals has been successfully interpreted in terms of the inductive and resonance effect of the substituents. Similarly, the locations of the anion states of aniline, phenol, anisole, and the halobenzenes may be explained ${ }^{19}$ in terms of these effects. However, alkyl groups were found ${ }^{11}$ to stabilize the benzene anion in the gas phase, contrary

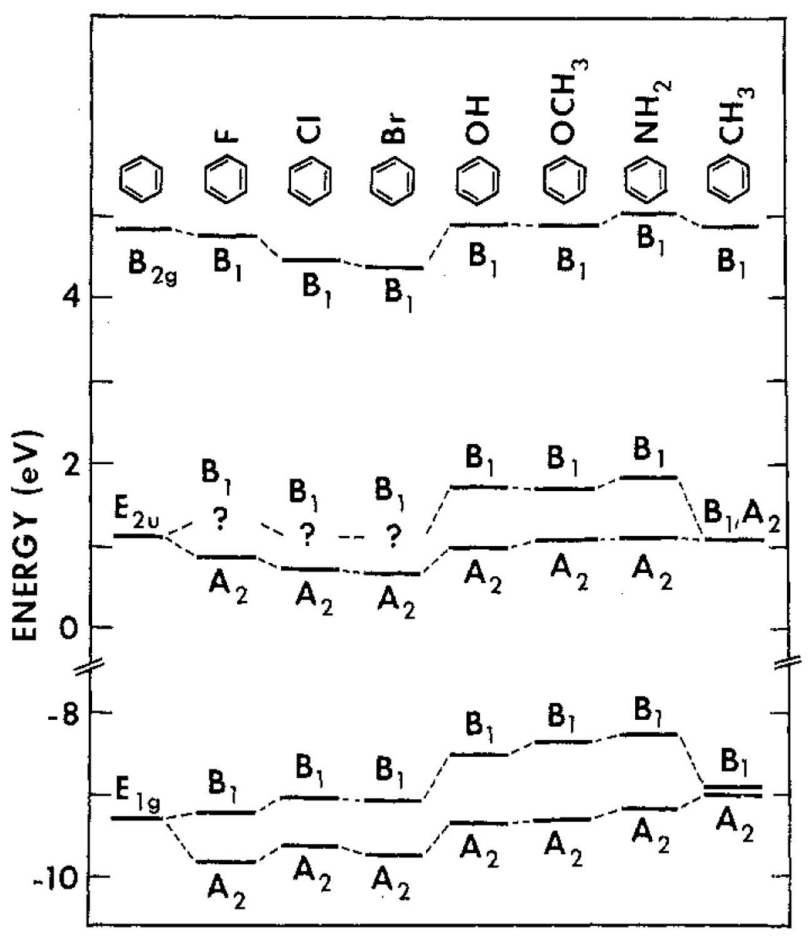

Figure 6. Correlation diagram using experimental values for the EAs and IPs of monosubstituted benzenes (from ref 19). 
to the results of solution studies by Lawler and Tabit ${ }^{20}$ and to predictions based on either resonance or inductive effects. Our findings are in agreement with recent studies by other researchers ${ }^{21}$ which demonstrated that in the gas phase $\mathrm{CH}_{3} \mathrm{O}^{-}$is more stable than $\mathrm{OH}^{-}$while in solution this order is reversed. Although this might lead to the speculation that alkyl groups always stabilize anions in the gas phase, we have also found that the anions of propene and acetaldehyde are less stable than those of ethylene and formaldehyde, respectively.

Perhaps the most interesting aspect revealed by these transmission studies is that the anions of the substituted benzenes mentioned above have shorter lifetimes than the benzene anion. For aniline, phenol, anisole, and the halobenzenes, the degenerate $\mathrm{e}_{2 \mathrm{u}}\left(\pi^{*}\right)$ orbital is split into $\mathrm{a}_{2}$ and $\mathrm{b}_{1}$ orbitals, with $\mathrm{a}_{2}$ lying below $\mathrm{b}_{1}$. While the $\mathrm{e}_{2 \mathrm{u}}$ orbital has a leading component of $l=3$, the $\mathrm{a}_{2}$ and $\mathrm{b}_{1}$ orbitals have $l$ $=2$ and 1 components, respectively. Even though the $a_{2}$ orbitals of these compounds have their dominant contribution from $l=3$, a simple model calculation shows ${ }^{22}$ that the mixing in of only a small percentage of the $l=2$ component causes significant changes in the lifetimes. Similarly, the presence of the $l=1$ and 2 components in the $b_{1}$ orbitals results in a complete loss of vibrational structure for the ${ }^{2} \mathrm{~B}_{1}$ anions. The negative ions formed by the occupation of the $\mathrm{a}_{2}$ orbital of these substituted benzenes are energetically more stable than the benzene anion by as much as $0.45 \mathrm{eV}$, but this increased stability is not sufficient to overcome the admixture of the $l=2$ component into the anions' charge distributions.

The ground-state anions of the alkyl-substituted benzenes are pseudo-Jahn-Teller systems ${ }^{23}$ since the Born-Oppenheimer splitting between the $A_{2}$ and $B_{1}$ states $^{24}$ is of the order of the energy associated with the zero-point nuclear motion. Therefore the vibronic ground states are admixtures of the $A_{2}$ and $B_{1}$ states and hence their charge distributions contain small contributions from $l=1$. For this reason, the anions of the alkylbenzenes have much weaker vibrational structure than does the benzene anion, even though the alkyl groups cause only a small shift in the EA.

Alternant Hydrocarbons. Alternant hydrocarbons have fascinated experimentalists and theorists for decades. Many of the unique features of these molecules have been explained in terms of the pairing theorem, which is a consequence of Hückel or PPP theories. 
Accordingly, the sum (IP + EA) associated with each $\pi, \pi^{*}$ pair should be constant. Although many papers examining the validity of the pairing theorem have appeared, usually only the first $\pi, \pi^{*}$ pair (i.e., HOMO, LUMO) has been considered, due to lack of accurate data for the higher lying negative ion states. ${ }^{25}$

We have used electron transmission to determine the EAs of several alternant hydrocarbons including ethylene, butadiene, hexatriene, benzene, styrene, stilbene, naphthalene, and cyclooctatetraene. ${ }^{26}$ In general, we have found that the pairing theorem holds remarkably well for those anion states which are predominantly single particle in nature $^{27}$ and which have sufficiently long lifetimes. For example, in styrene, the sum (IP + EA) yields 8.18, 8.13, and 7,97 eV for the first three $\pi, \pi^{*}$ pairs. This is most encouraging since it implies that for these compounds one can predict, from the appropriate IPs, the electron affinities associated with stable anions which cannot be determined directly by electron transmission.

In Figure 7 we present a correlation diagram ${ }^{28}$ using experimentally determined energies for the cation and anion states of styrene as well as those of ethylene and benzene. It is readily apparent from this diagram that one can interpret the four negative ion states of styrene as arising from the interaction of anion states of ethylene and benzene. It is reassuring to note that concepts such as the pairing theorem and the description of a composite system in terms of its interacting moieties $^{29}$ retain their utility in dealing with these short-lived species.

We emphasize again that electron transmission spectra provide more than just the energies of the anion states. As an illustration, the spectra of styrene and benzene are shown in Figure 8. A wide range of anion lifetimes are evident, and the relative values can be understood in terms of the anion energies and symmetries, ${ }^{22}$ although space does not permit a discussion here. A detailed analysis of the vibrational structure, which has not yet been carried out, should also yield useful information concerning the geometries of the anions.

\section{Applications to Other Chemical Studies}

Molecular Orbital Theories of Reactivity. The various molecular orbital theories of reactivity employ information about the occupied and unoccupied orbitals of the interacting systems to predict reaction 


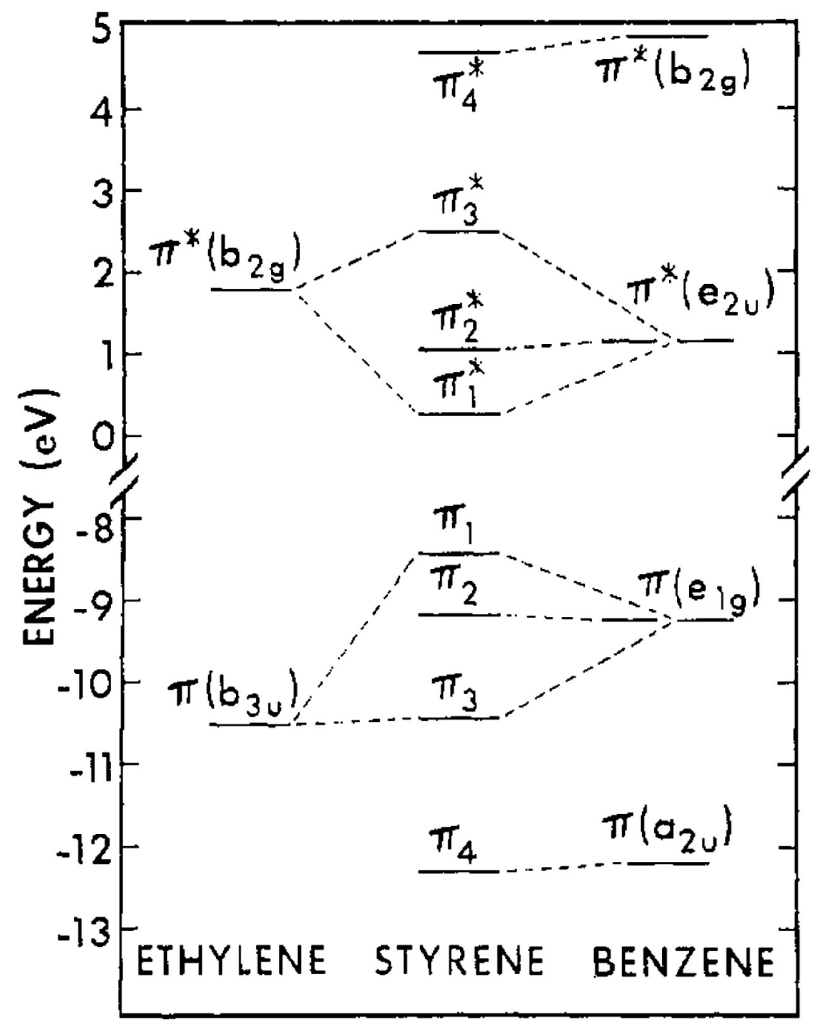

Figure 7. Correlation diagram using experimental values for the EAs and IPs of ethylene, styrene, and benzene (from ref 28).

rates. One of the most useful of these is the frontier molecular orbital method ${ }^{30}$ which utilizes the energies and shapes of the HOMO and LUMO to determine the reactivity of a molecule towards electrophiles or nucleophiles, respectively. This approach has proven particularly useful in describing a wide variety of cycloaddition reactions. ${ }^{31}$ However, it has often been necessary to employ estimated EAs due to the paucity of experimental values. The availability of accurate experimental values of the EAs for a wide range of hydrocarbons will allow for a stringent test of the theory and facilitate its application.

Singlet and Triplet Excitation Energies. To a good approximation, the low-lying excitation energies for a series of related compounds may often be correlated to the quantity (IP - EA). ${ }^{32}$ The usefulness of such a correlation is illustrated in Figure 9 where we have plotted the lowest triplet $\left(\mathrm{T}_{1}\right)$ and singlet $\left(\mathrm{S}_{1}\right)$ excitation energies vs. (IP $\mathrm{POMO}_{\text {Hо }}$ - $\mathrm{EA}_{\mathrm{LUMO}}$ ) for a number of monosubstituted benzenes. ${ }^{33}$ Both the $\mathrm{T}_{1}$ 


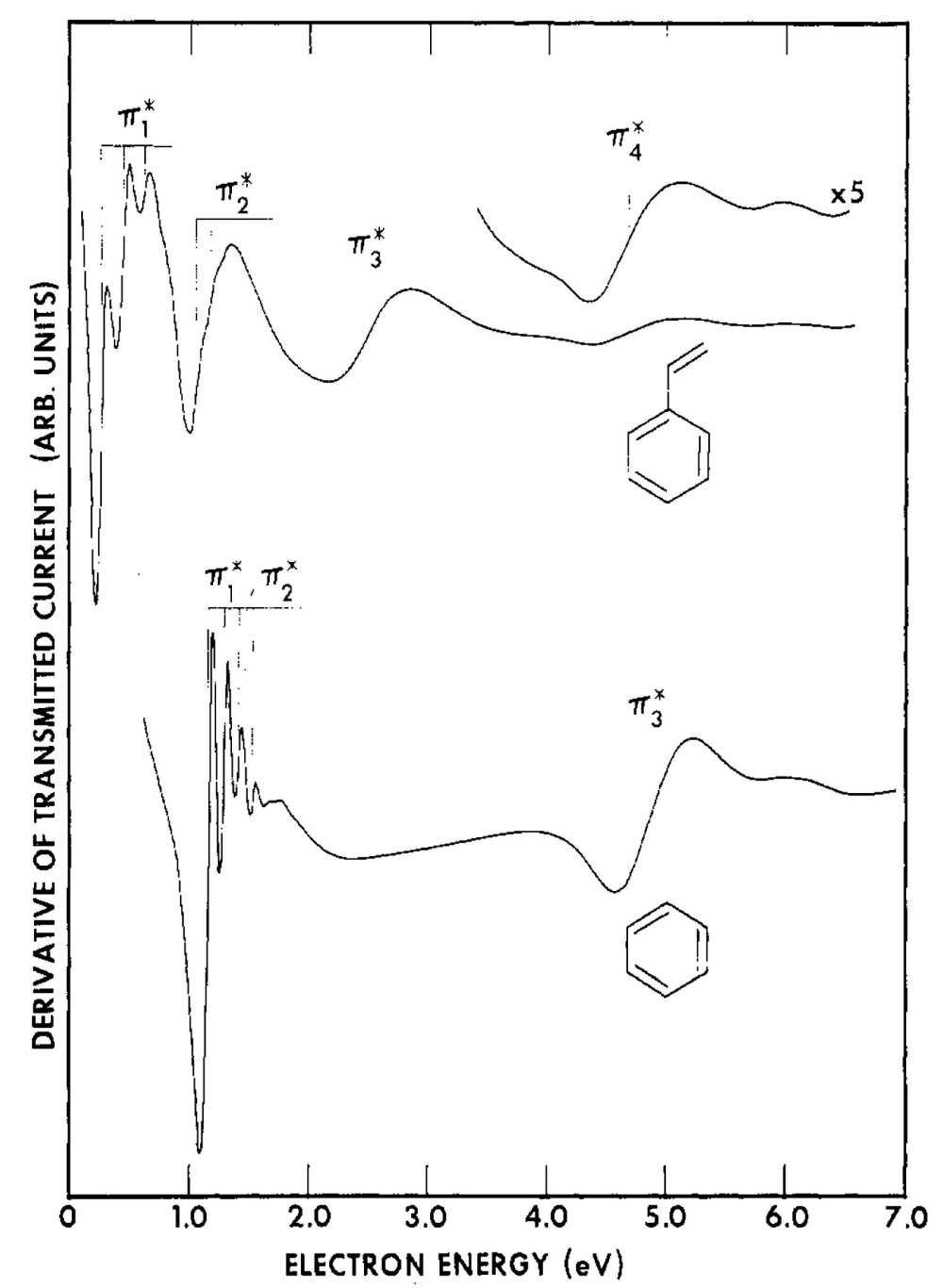

Figure 8. The derivative of transmitted current as a function of electron energy in styrene (upper) and benzene (lower) (from ref 28).

and $\mathrm{S}_{1}$ excitation energies for this series of components are nearly linear functions of ( $\mathrm{IP}_{\text {Hомо }}-\mathrm{EA}_{\mathrm{LUMO}}$ ), with slopes of 0.21 and 0.27 , respectively. Using perturbation theory it may be shown that the singlet excitation energy is equal to $\left(\mathrm{IP}_{\mathrm{HOMO}}-\mathrm{EA}_{\mathrm{LUMO}}\right)+$ electron-electron interaction terms), where the two-electron interaction terms include the first-order Coulomb and exchange matrix elements as well as second and higher order correlation terms. The results displayed in Figure 9 indicate that the two-electron terms are approximately linearly related to (IP - EA). The linear relationship between the $\mathrm{T}_{1}$ excitation energies and ( $\left.\mathrm{IP}_{\mathrm{HOMO}}-\mathrm{EA}_{\mathrm{LUMO}}\right)$ is surprising since for the substituted 


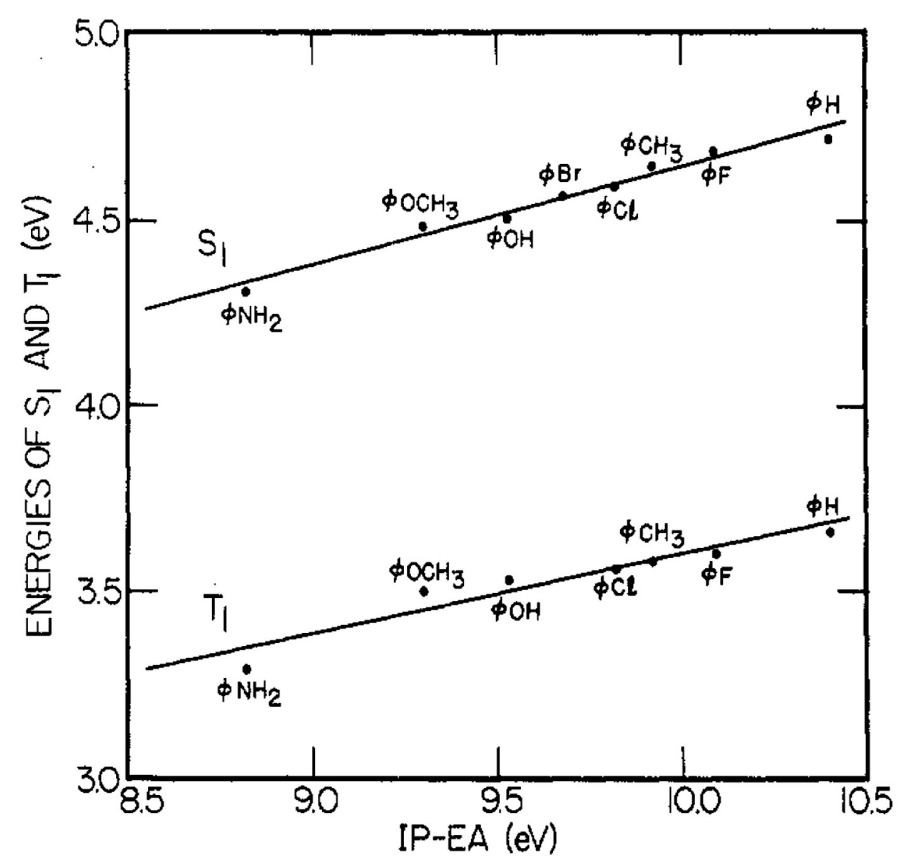

Figure 9. The lowest singlet, $\mathrm{S}_{1}$, and triplet, $\mathrm{T}_{1}$, transition energies of several monosubstituted benzenes as a function of $\mathrm{IP}_{\mathrm{HOMO}}-\mathrm{EA}_{\mathrm{LUMO}}$.

benzenes these transitions are not described by an electron promoted from $\mathrm{HOMO}$ to LUMO. Rather, the $\mathrm{T}_{1}$ transitions involve a mixing between the excitation from the HOMO to the second unoccupied orbital and the excitation from the second occupied orbital to the LUMO.

Anions in Solution. The ground-state anions of most unsaturated hydrocarbons can be prepared as stable species in solution or in hydrocarbon glasses by alkali reduction or by means of gamma radiation. ${ }^{34}$ Numerous studies of the absorption spectra of these stable ions have been carried out. A detailed comparison between the condensed-phase excitation energies and the gas phase values, determined by subtracting from the first EA the higher EAs of a molecule, will yield valuable information concerning solvation effects.

For styrene and naphthalene, we have found that the agreement is within $0.15 \mathrm{eV}$ for those excitations predominantly involving transitions between $\pi^{*}$ orbitals and for which the gas-phase anions are sufficiently long-lived. The agreement is poorer for those transitions to very short-lived anion states, indicating that solvation effects are quite 
different for these anion states. We note that certain transitions may not be seen in the condensed-phase studies if they are dipole-forbidden or only weakly allowed. The electron transmission method does not suffer from this limitation. It may happen, however, that an anion which is relatively long-lived in solution may have such a short lifetime in the gas phase that its energy cannot be determined.

\section{Conclusions}

In this account, we have described the electron transmission technique and illustrated a few of the areas which will benefit from its application to hydrocarbon molecules. Without doubt the most important data resulting from transmission studies are the temporary anion energies. As a convenient reference, a table of the values which we regard as being most reliable is included. A complete bibliography of published work using electron transmission is available from one of the authors (P.D.B.).

We have also emphasized the close connection between orbital symmetry and anion lifetime and have pointed out the sensitivity of lifetime to substituents. This is an area which deserves detailed theoretical exploration.

Space has permitted only a discussion of those anions associated with the capture of electrons into vacant $\pi *$ orbitals. Other anion states, with two electrons in Rydberg orbitals, are also known to exist in some hydrocarbons. For most molecules the $\sigma^{*}$ orbitals are sufficiently high in energy that the corresponding anions are too shortlived to be detected in transmission. We anticipate, however, that these states will be evident as broad enhancements of the cross sections for vibrational excitation of the neutral molecule by electron impact.

Acknowledgments This paper is respectfully dedicated to the late George J. Schulz, whose pioneering contributions to the field of temporary anions made this work possible. We gratefully acknowledge the help of our colleague J. A. Michejda. Part of this article was written while K.D.J. was a Visiting Scholar at the University of Nebraska. 
Table I Vertical Electron Affinities (eV) Determined by Electron Transmission Spectroscopy

\begin{tabular}{|c|c|}
\hline ethylene $^{a}$ & -1.78 \\
\hline propene $^{b}$ & -1.99 \\
\hline cis-butene ${ }^{b}$ & -2.22 \\
\hline trans-butene $\mathrm{e}^{c}$ & -2.10 \\
\hline isobutene $^{c}$ & -2.19 \\
\hline trimethylethylene $e^{e}$ & -2.24 \\
\hline tetramethylethylene $e^{c}$ & -2.27 \\
\hline cyclohexene $^{b}$ & -2.07 \\
\hline formaldehyde $e^{d, e}$ & -0.86 \\
\hline acetaldehyde ${ }^{c, e}$ & -1.19 \\
\hline acetone $^{c, e}$ & -1.51 \\
\hline acetylene $e^{f}$ & -2.6 \\
\hline acetonitrile $f$ & -2.84 \\
\hline acrylonitrile ${ }^{f}$ & $-0.21,-2.74$ \\
\hline 1,3-butadiene ${ }^{a}$ & $-0.62,-2.8$ \\
\hline 1,3 -cyclohexadiene ${ }^{b}$ & $-0.80,-3.43$ \\
\hline 1,4 -cyclohexadiene ${ }^{b}$ & $-1.75,-2.67$ \\
\hline 1,5 -cyclooctadiene ${ }^{b}$ & $-1.83,-2.33$ \\
\hline norbornadiene $e^{b}$ & $-1.04,-2.56$ \\
\hline benzene ${ }^{g, h}$ & $-1.15,-4.85$ \\
\hline toluene $^{g}$ & $-1.11,-4.88$ \\
\hline ethylbenzene $^{i}$ & -1.17 \\
\hline isopropylbenzene $^{g}$ & $-1.08,-4.69$ \\
\hline terf-butylbenzene ${ }^{g}$ & $-1.06,-4.67$ \\
\hline cyclopropylbenzene ${ }^{f}$ & $-1.06,-4.59$ \\
\hline$o$-xylene ${ }^{g}$ & $-1.12,-4.9$ \\
\hline$m$-xylene ${ }^{g}$ & $-1.06,-4.81$ \\
\hline$p$-xylene ${ }^{g}$ & $-1.07,-4.89$ \\
\hline 1,3,5-trimethylbenzene ${ }^{g}$ & $-1.03,-4.78$ \\
\hline 1,2,4-trimethylbenzene ${ }^{g}$ & $-1.07,-4.83$ \\
\hline phenoj & $-1.01,-1.73,-4.92$ \\
\hline anisole $^{j}$ & $-1.09,-1.72,-4.92$ \\
\hline aniline $^{j}$ & $-1.13,-1.85,-5.07$ \\
\hline methylaniline ${ }^{f}$ & -1.19 \\
\hline dimethylaniline $^{f}$ & -1.24 \\
\hline fluorobenzene $^{j}$ & $-0.89,-4.77$ \\
\hline chlorobenzene $^{j}$ & $-0.75,-4.50$ \\
\hline bromobenzene $^{j}$ & $-0.70,-4.42$ \\
\hline pyridine $^{k}$ & $-0.62,-1.20,-4.58$ \\
\hline pyrimidine $^{k}$ & $>0,-0.77,-4.24$ \\
\hline pyridazine $^{k}$ & $>0,-0.73,-4.05$ \\
\hline$s$-triazine ${ }^{k}$ & $>0,-4.0$ \\
\hline
\end{tabular}


Table I Vertical Electron Affinities (eV) Determined by Electron Transmission Spectroscopy (continued)

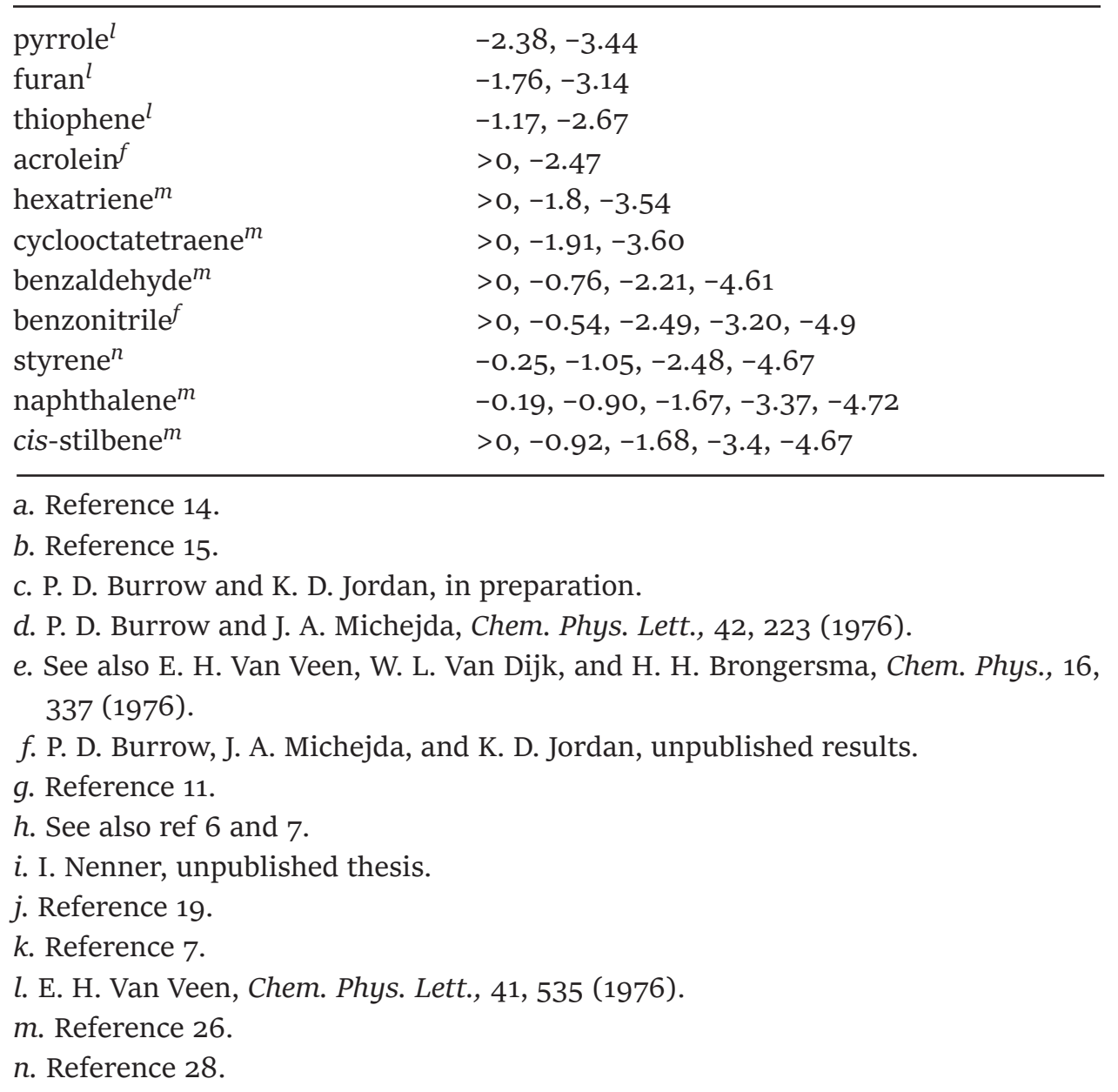

\section{References}

(1) G. J. Schulz, Rev. Mod. Phys., 45, 378, 423 (1973).

(2) L. Sanche and G. J. Schulz, Phys. Rev. A, 5, 1672 (1972).

(3) P. D. Burrow, J. A. Michejda, and J. Comer, J. Phys. B, 9, 3225 (1976).

(4) F. H. Read, J. Phys. B, 1, 893 (1968); D. Andrick and F. H. Read, ibid., 4, 389 (1971).

(5) M. J. W. Boness, I. W. Larkin, J. B. Hasted, and L. Moore, Chem. Phys. Lett., 1, 292 (1967); D. Mathur and J. B. Hasted, Chem. Phys., 16, 347 (1976).

(6) L. Sanche and G. J. Schulz, Phys. Rev. Lett., 26, 943 (1971); ibid., 27, 1333 (1971); Phys. Rev. A, 6, 69, 2500 (1972); J. Chem. Phys., 58, 479 (1973).

(7) I. Nenner and G. J. Schulz, J. Chem. Phys., 62, 1747 (1975).

(8) That is, the period which would result if the anion had infinite lifetime.

(9) D. T. Birtwistle and A. Herzenberg, J. Phys. B, 4, 53 (1971). 
(10) J. N. Bardsley and F. Mandl, Rep. Prog. Phys., 31, 471 (1968).

(11) K. D. Jordan, J. A, Michejda, and P. D. Burrow, J. Am. Chem. Soc., 98, 1295 (1976).

(12) G. J. Schulz, "Principles of Laser Plasmas", Bekefi, Ed., Wiley, New York, N.Y., 1976, p 33 .

(13) S. F. Wong and G. J. Schulz, Phys. Rev. Lett., 35, 1429 (1975).

(14) P. D. Burrow and K. D. Jordan, Chem. Phys. Lett., 36, 594 (1975).

(15) K. D. Jordan, J. A. Michejda, and P. D. Burrow, Chem. Phys. Lett., 42, 227 (1976).

(16) R. Hoffmann, Acc. Chem. Res., 4, 1 (1971).

(17) C. Batich, P. Bischof, and E. Heilbronner, J. Electron Spectrosc. Relat. Phenom., 1, 333 (1972).

(18) R. Hoffmann, E. Heilbronner, and R. Gleiter, J. Am. Chem. Soc., 92, 706 (1970).

(19) K. D. Jordan, J. A. Michejda, and P. D. Burrow, J. Am. Chem. Soc., 98, 7189 (1976).

(20) R. G. Lawler and C. T. Tabit, J. Am. Chem. Soc., 91, 5671 (1969).

(21) J. I. Brauman and L. K. Blair, J. Am. Chem. Soc., 92, 5986 (1970), and earlier works; M. J. McAdams and L. I. Bone, J. Phys. Chem., 75, 2226 (1971); R. Yamdagni and P. Kebarle, J. Am. Chem. Soc., 95, 4050 (1973).

(22) K. D. Jordan and P. D. Burrow, in preparation.

(23) J. S. Alper and R. J. Silbey, J. Chem. Phys., 52, 569 (1970); D. Purina and M. Karplus, J. Am. Chem. Soc., 9o, 6275 (1968).

(24) Here we employ the $C_{2 v}$ point group to label the states of the alkylbenzenes.

(25) Recently, MCD spectroscopy has proven to be a very useful tool for studying the validity of the pairing theorem. See, for example, J. Michl, J. Chem. Phys., 61, 4270 (1974); Chem. Phys. Lett., 43, 457 (1976).

(26) P. D. Burrow, J. A. Michejda, and K. D. Jordan, in preparation.

(27) As opposed to those states which are two-particle, one-hole; that is, a bound electron is promoted from $\pi$ to $\pi^{*}$ and the incident electron occupies a $\pi^{*}$ orbital.

(28) P. D. Burrow, J. A. Michejda, and K. D. Jordan, J. Am. Chem. Soc., 98, 6392 (1976).

(29) W. F. Frey, R. N. Compton, W. T. Naff, and H. C. Schweinler, Int. J. Mass Spectrom. Ion Phys., 12, 19 (1973).

(30) See, for example, K. N. Houk and L. L. Munchausen, J. Am. Chem. Soc., 98, 937 (1976).

(31) K. N. Houk, Acc. Chem. Res., 8, 361 (1975).

(32) See, for example, J. Michl and R. S. Becker, J. Chem. Phys., 46, 3889 (1967).

(33) A similar plot was presented in the unpublished thesis of I. Nenner (Universite de Paris-Sud, 1975). We have replaced Nenner's EAs with our own values which we believe to be slightly more accurate.

(34) T. Shida and S. Iwata, J. Am. Chem. Soc., 95, 3473 (1973). 


\section{About the authors}

Kenneth D. Jordan was born in Norwood, Mass., in 1948. He studied at Northeastern University for his undergraduate degree and went on to graduate work at Massachusetts Institute of Technology, where he received the Ph.D. Dr. Jordan is Assistant Professor at Yale University and an Alfred P. Sloan Foundation Fellow (1977-1979). His research interests include bound and temporary anions of hydrocarbons, electron-polar molecule interactions, the electronic structure of metal clusters, and reaction mechanisms.

Paul D. Burrow is Associate Professor of Physics at the University of Nebraska. He was born in Oklahoma City in 1938, received an S.B. degree from MIT, and the Ph.D. from University of California, Berkeley. Before moving to Nebraska in 1976, Dr. Burrow taught at Yale University. His research is concerned with temporary anions of atoms and molecules and scattering processes between electrons and excited species. 